\title{
Disentangling Strong-Field Multielectron Dynamics with Angular Streaking
}

Alexander H. Winney, Gihan Basnayake (iD), Duke A. Debrah (D), Yun Fei Lin, Suk Kyoung Lee, Paul Hoerner, Qing Liao, $\underline{\text { H. Bernhard Schlegel }}$ (iD), and Wen Li

Department of Chemistry, Wayne State University, Detroit, Michigan 48202, United States

J. Phys. Chem. Lett., 2018, 9 (10), pp 2539-2545

DOI: https://pubs.acs.org/doi/10.1021/acs.jpclett.8b00028

\section{Tracking Ultrafast Bond Dissociation Dynamics at $0.1 \AA$ Resolution by Femtosecond Extreme Ultraviolet Absorption Spectroscopy}

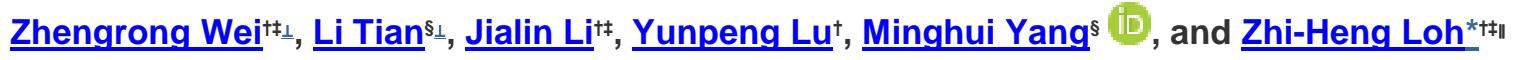

† Division of Chemistry and Biological Chemistry, School of Physical and Mathematical

Sciences, Nanyang Technological University, Singapore 637371

‡ Division of Physics and Applied Physics, School of Physical and Mathematical Sciences, Nanyang

Technological University, Singapore 637371

s Key Laboratory of Magnetic Resonance in Biological Systems, State Key Laboratory of Magnetic Resonance and Atomic and Molecular Physics, National Center for Magnetic Resonance in Wuhan, Wuhan Institute of Physics and Mathematics, Chinese Academy of Sciences, Wuhan 430071, China " Centre for Optical Fibre Technology, The Photonics Institute, Nanyang Technological University, Singapore 639798

J. Phys. Chem. Lett., 2018, 9 (19), pp 5742-5747

DOI: https://pubs.acs.org/doi/10.1021/acs.jpclett.8b02547

\section{Direct Signatures of Light-Induced Conical Intersections on the Field-Dressed Spectrum of $\mathrm{Na}_{2}$}

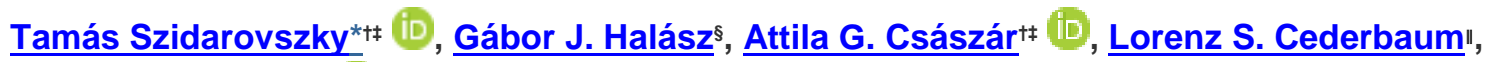
and Ágnes Vibók ${ }^{\star_{\perp} \#}$ (iD

+ Laboratory of Molecular Structure and Dynamics, Institute of Chemistry, Eötvös Loránd University, P.O.

Box 32, H-1117Budapest, Hungary

‡ MTA-ELTE Complex Chemical Systems Research Group, Pázmány Péter sétány 1/A, H-

1117 Budapest, Hungary

s Department of Information Technology, University of Debrecen, P.O. Box 400, H-

4002 Debrecen, Hungary

" Theoretische Chemie, Physikalisch-Chemisches Institut, Universität Heidelberg, D-

69120 Heidelberg, Germany 
$\perp$ Department of Theoretical Physics, University of Debrecen, P.O. Box 400, H-4002 Debrecen, Hungary

\# ELI-ALPS, ELI-HU Non-Profit Ltd., Dugonics tér 13, H-6720 Szeged, Hungary

J. Phys. Chem. Lett., 2018, 9 (11), pp 2739-2745

DOI: https://pubs.acs.org/doi/10.1021/acs.jpclett.8b01102

\section{Coherent Control of the Rotation Axis of Molecular Superrotors}

$\underline{\text { A. Owens }}^{\dagger \neq}, \underline{\text { A. Yachmenev }}^{\star \neq \ddagger}$ (D), and J. Küpper ${ }^{\dagger+\$}$

+ Center for Free-Electron Laser Science, Deutsches Elektronen-Synchrotron DESY, Notkestraße

85, 22607 Hamburg, Germany

‡ The Hamburg Center for Ultrafast Imaging, Universität Hamburg, Luruper Chaussee

149, 22761 Hamburg, Germany

\& Department of Physics, Universität Hamburg, Luruper Chaussee 149, 22761 Hamburg, Germany

J. Phys. Chem. Lett., 2018, 9 (15), pp 4206-4209

DOI: https://pubs.acs.org/doi/10.1021/acs.jpclett.8b01689

\section{Nuclear Motion Driven Ultrafast Photodissociative Charge Transfer of the PENNA Cation: An Experimental and Computational Study}

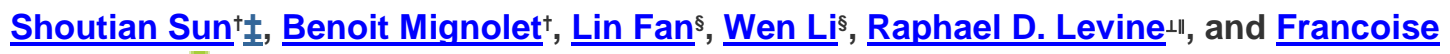

$\underline{\text { Remacle }}^{+}$

† Department of Chemistry, B6c, University of Liege, B4000 Liege, Belgium

$\S$ Department of Chemistry, Wayne State University, Detroit, Michigan 48202, United States

${ }_{\perp}$ Crump Institute for Molecular Imaging and Department of Molecular and Medical Pharmacology, David Geffen School of Medicine, and Department of Chemistry and Biochemistry, University of California, Los Angeles, California 90095, United States

" The Fritz Haber Research Center for Molecular Dynamics, Institute of Chemistry, The Hebrew University of Jerusalem, 91904 Jerusalem, Israel

J. Phys. Chem. A, 2017, 121 (7), pp 1442-1447

DOI: https://pubs.acs.org/doi/10.1021/acs.jpca.6b12310

\section{Identifying Strong-Field Effects in Indirect Photofragmentation Reactions}

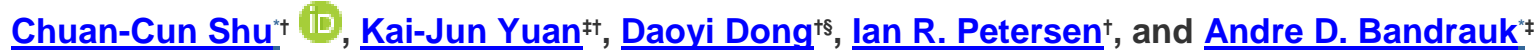

+ School of Engineering and Information Technology, University of New South Wales, Canberra, Australian Capital Territory 2600, Australia 
‡ Laboratoire de Chimie Théorique, Faculté des Sciences, Université de Sherbrooke Sherbrooke, Québec J1K 2R1, Canada

$\S$ Department of Chemistry, Princeton University, Princeton, New Jersey 08544, United States

J. Phys. Chem. Lett., 2017, 8 (1), pp 1-6

DOI: https://pubs.acs.org/doi/10.1021/acs.jpclett.6b02613

\section{Angular Dependence of Strong Field Ionization of Haloacetylenes $\operatorname{HCCX}(\mathrm{X}=\mathrm{F}, \mathrm{Cl}, \mathrm{Br}, \mathrm{I})$, Using Time-Dependent Configuration Interaction with an Absorbing Potential}

$\underline{\text { Paul Hoerner and } \underline{\mathrm{H} . \text { Bernhard Schlegel }}}{ }^{*}$

Department of Chemistry, Wayne State University, Detroit, Michigan 48202, United States

J. Phys. Chem. C, 2018, 122 (25), pp 13751-13757

DOI: https://pubs.acs.org/doi/10.1021/acs.jpcc.8b00619

\section{Molecular Double Ionization Using Strong Field Few-Cycle Laser Pulses}

$\underline{\text { Arthur Zhao }}^{\dagger}$, Péter Sándor $^{\dagger}$, Vincent Tagliamonti $^{\dagger}$, Spiridoula Matsika $^{\ddagger}$, and Thomas Weinacht $^{\dagger}$ + Department of Physics and Astronomy, Stony Brook University, Stony Brook, New York 117943800, United States

‡ Department of Chemistry, Temple University, Philadelphia, Pennsylvania 19122, United States J. Phys. Chem. A, 2016, 120 (19), pp 3233-3240

DOI: https://pubs.acs.org/doi/10.1021/acs.jpca.5b11713

\section{Order of Magnitude Dissociative Ionization Enhancement Observed for Pulses with High Order Dispersion}

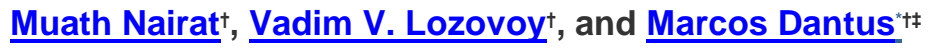

† Department of Chemistry, Michigan State University, East Lansing, Michigan 48824, United States

‡ Department of Physics and Astronomy, Michigan State University, East Lansing, Michigan

48824, United States

J. Phys. Chem. A, 2016, 120 (43), pp 8529-8536

DOI: https://pubs.acs.org/doi/10.1021/acs.jpca.6b08659 


\title{
Probing in Space and Time the Nuclear Motion Driven by Nonequilibrium Electronic Dynamics in Ultrafast Pumped $\mathrm{N}_{2}$
}

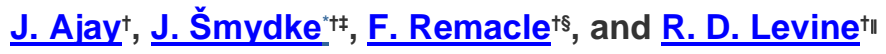 \\ † The Fritz Haber Center for Molecular Dynamics and Institute of Chemistry, The Hebrew University of \\ Jerusalem, Jerusalem 91904, Israel \\ ‡ Department of Radiation and Chemical Physics, Institute of Physics, Academy of Sciences of the Czech \\ Republic, 18221 Praha 8, Czech Republic \\ $\S$ Département de Chimie, B6c, Université de Liège, B4000 Liège, Belgium \\ " Crump Institute for Molecular Imaging and Department of Molecular and Medical Pharmacology, David \\ Geffen School of Medicine and Department of Chemistry and Biochemistry, University of California, Los \\ Angeles, California 90095, United States
}

J. Phys. Chem. A, 2016, 120 (19), pp 3335-3342

DOI: https://pubs.acs.org/doi/10.1021/acs.jpca.6b00165

\section{Attosecond Charge Migration with TDDFT: Accurate Dynamics from a Well-Defined Initial State}

$\underline{\text { Adam Bruner }}^{\dagger}\left(\right.$ D) Samuel Hernandez $^{\dagger}$, Francois Mauger $^{\ddagger}$, Paul M. Abanador $^{\ddagger}$, Daniel J.

LaMaster $^{\dagger}$, Mette B. Gaarde $^{\ddagger}$, Kenneth J. Schafer $^{\ddagger}$, and Kenneth Lopata ${ }^{\dagger}+\$$

+ Department of Chemistry, Louisiana State University, Baton Rouge, Louisiana 70803, United States

‡ Department of Physics and Astronomy, Louisiana State University, Baton Rouge, Louisiana

70803, United States

\& Center for Computation \& Technology, Louisiana State University, Baton Rouge, Louisiana

70803, United States

J. Phys. Chem. Lett., 2017, 8 (17), pp 3991-3996

DOI: https://pubs.acs.org/doi/10.1021/acs.jpclett.7b01652

\section{Wavelength Dependence of the Suppressed Ionization of Molecules in Strong Laser Fields}

$\underline{\text { J. Durá*}} \dagger, \underline{A}$. Grünt, P. K. Batest, S. M. Teichmann†, T. Erglert, A. Senftlebent, T. Pflügerł, C. D.

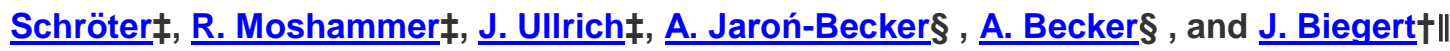

† ICFO-Institut de Ciencies Fotoniques, Av. Carl Friedrich Gauss 3, 08860 Castelldefels (Barcelona), Spain

‡ Max-Planck-Institut für Kernphysik, Saupfercheckweg 1, 69117 Heidelberg, Germany

$\S \mathrm{JILA}$ and Department of Physics, University of Colorado, UCB 440, Boulder 80309-0440, United States

" ICREA-Institucio Catalana de Recerca i Estudis Avançats, 08010 Barcelona, Spain

J. Phys. Chem. A, 2012, 116 (11), pp 2662-2668 
DOI: $\underline{\text { https://pubs.acs.org/doi/10.1021/ip207257i }}$

\section{Elucidating Strong Field Photochemical Reduction Mechanisms of Aqueous [AuCl 4$]^{-}:$Kinetics of Multiphoton Photolysis and Radical-Mediated Reduction}

$\underline{\text { Katharine Moore Tibbetts }}^{\dagger}$, Behzad Tangeysh $^{\dagger}$, Johanan H. Odhner ${ }^{\dagger}$, and Robert J. Levis ${ }^{\dagger}$

† Department of Chemistry and Center for Advanced Photonics Research, Temple University,

Philadelphia, Pennsylvania 19122, United States

J. Phys. Chem. A, 2016, 120 (20), pp 3562-3569

DOI: https://pubs.acs.org/doi/10.1021/acs.jpca.6b03163

\section{State-Specific Collision Dynamics of Molecular Super Rotors with Oriented Angular Momentum}

Matthew J. Murray, Hannah M. Ogden, Carlos Toro, Qingnan Liu, David A. Burns, Millard H. Alexander, and Amy S. Mullin

Department of Chemistry and Biochemistry, University of Maryland, College Park, Maryland 20742, United States

J. Phys. Chem. A, 2015, 119 (50), pp 12471-12479

DOI: https://pubs.acs.org/doi/10.1021/acs.jpca.5b07941

\section{Gaining Mechanistic Insight with Control Pulse Slicing: Application to the Dissociative Ionization of $\mathrm{CH}_{2} \mathrm{Brl}$}

Xi Xing, Roberto Rey-de-Castro, and Herschel Rabitz

Department of Chemistry, Princeton University, Princeton, New Jersey 08544, United States

J. Phys. Chem. A, 2017, 121 (45), pp 8632-8641

DOI: https://pubs.acs.org/doi/10.1021/acs.jpca.7b08835

\section{Intense Laser Alignment As a Route to Control of Surface Reactions}

$\underline{\text { Deepika Shreenivas }^{\dagger}}, \underline{\text { Anthony Lee }^{\dagger}}, \underline{\text { Nadine Walter }}{ }^{\ddagger}, \underline{\text { David Sampayo }}, \underline{\text { Steve Bennett }}{ }^{\dagger}$ and $\underline{\text { Tamar }}$ $\underline{\text { Seideman* }}^{*}$

Department of Chemistry, Northwestern University, 2145 Sheridan Road, Evanston, Illinois 60208-3113 


\title{
Effect of Laser Parameters on Ultrafast Hydrogen Migration in Methanol Studied by Coincidence Momentum Imaging
}

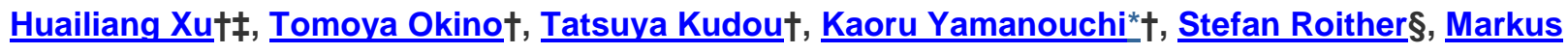 \\ Kitzler§, Andrius Baltuska§, and See-Leang Chin $\perp$ \\ † Department of Chemistry, School of Science, The University of Tokyo, 7-3-1 Hongo, Bunkyo-ku, Tokyo \\ 113-0033, Japan \\ ‡ State Key Laboratory on Integrated Optoelectronics, College of Electronic Science and Engineering, Jilin \\ University, Changchun 130012, China \\ \& Photonics Institute, Vienna University of Technology, Gusshausstrasse 27, A-1040 Vienna, Austria \\ ${ }_{\perp}$ Center for Optics, Photonics and Laser (COPL) \& Department of Physics, Engineering Physics and \\ Optics, Laval University, Quebec City, QC, Canada G1 V OA6 \\ J. Phys. Chem. A, 2012, 116 (11), pp 2686-2690 \\ DOI: https://pubs.acs.org/doi/10.1021/jp207483y
}

\section{Transition from SAMO to Rydberg State Ionization in $\mathrm{C}_{60}$ in Femtosecond Laser Fields}

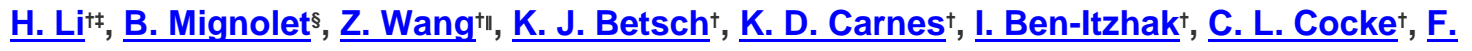
Remacle $^{*}$, and M. F. Kling ${ }^{+\perp}$

† J. R. Macdonald Laboratory, Physics Department, Kansas State University, Manhattan, Kansas 66506, United States

‡ State Key Laboratory of Precision Spectroscopy, East China Normal University, Shanghai 200062, China

$\S$ Department of Chemistry, University of Liege, B-4000 Liege, Belgium

" The MOE Key Laboratory of Weak-Light Nonlinear Photonics, TEDA Applied Physics Institute and School of Physics, Nankai University, Tianjin 300457, China

${ }_{\perp}$ Department of Physics, Ludwig-Maximilians-Universität Munich, D-85748 Garching, Germany J. Phys. Chem. Lett., 2016, 7 (22), pp 4677-4682

DOI: $\underline{\text { https://pubs.acs.org/doi/10.1021/acs.jpclett.6b02139 }}$

\section{A Stark Future for Quantum Control}

\author{
$\underline{\text { Dave Townsend }}^{\star \dagger}$, Benjamin J. Sussman $^{\ddagger}$, and Albert Stolow ${ }^{\ddagger}$
}


School of Engineering and Physical Sciences, Heriot-Watt University, Edinburgh, EH14 4AS, United Kingdom, and Steacie Institute for Molecular Sciences, National Research Council of Canada, 100 Sussex Drive, Ottawa, Ontario, K1A 0R6, Canada

J. Phys. Chem. A, 2011, 115 (4), pp 357-373

DOI: https://pubs.acs.org/doi/10.1021/ip109095d

\title{
Coulomb Explosion Imaging for the Visualization of a Conical Intersection
}

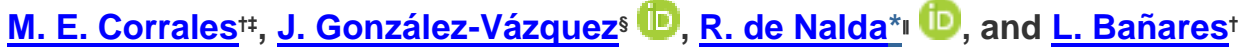 \\ + Departamento de Química Física (Unidad Asociada I+D+i al CSIC), Facultad de Ciencias \\ Químicas, Universidad Complutense de Madrid, 28040 Madrid, Spain \\ ₹ Centro de Láseres Ultrarrápidos, Facultad de Ciencias Químicas, Universidad Complutense de \\ Madrid, 28040 Madrid, Spain \\ § Departamento de Química and Institute for Advanced Research in Chemical Sciences (IAdChem), \\ Módulo 13, Facultad de Ciencias, Universidad Autónoma de Madrid, 28049 Madrid, Spain \\ " Instituto de Química Física Rocasolano, CSIC, C/Serrano 119, 28006 Madrid, Spain \\ J. Phys. Chem. Lett., 2019, 10 (2), pp 138-143
}

DOI: https://pubs.acs.org/doi/10.1021/acs.jpclett.8b03726 